\title{
Wer kontrolliert die Kontrolleure? Zehn Thesen zum sogenannten Impact Factor
}

\author{
W. Golder \\ Abteilung für Radiologische Diagnostik und Nuklearmedizin, \\ Klinikum Benjamin Franklin, Freie Universität Berlin
}

Schlüsselwörter

Impact Factor · Quellenzeitschrift · Szientometrie · Zitat

\section{Zusammenfassung}

Der Impact Factor hat sich zu einem vielverwendeten Instrument bei der Bewertung wissenschaftlicher Arbeit entwickelt. Er ist definiert als Quotient aus der Zahl der in den beiden letzten Jahren erschienenen Zitate von Artikeln einer Zeitschrift und der Zahl der dort im gleichen Zeitraum publizierten Aufsätze. Bei der Verwendung des Parameters sollte man die vielen Faktoren nicht vernachlässigen, die das Zitierverhalten beeinflussen. Die Korrelation zwischen dem Impact Factor einer Zeitschrift und der Häufigkeit, mit der ein dort veröffentlichter Aufsatz zitiert wird, ist fragwürdig. Die Auswahl von Zitaten unterliegt qualitätsunabhängigen Faktoren. Englischsprachige Zeitschriften werden viel häufiger zitiert als solche, die in anderen Sprachen erscheinen. Der Impact Factor begünstigt Arbeitsgebiete, die kurzzeitig gültige Resultate liefern, und benachteiligt langfristige klinische Forschungen.

\section{Einleitung}

Der sogenannte Impact Factor (IF), der vom Institute for Scientific Information in Philadelphia, PA, berechnet und vermarktet wird, hat in den letzten Jahren überragende Bedeutung für das Ansehen und die Konjunktur akademischer Periodika und der dort publizierenden Wissenschaftler gewonnen. Er liefert ein Maß für die Häufigkeit, mit der der durchschnittliche Artikel einer Zeitschrift in den beiden dem laufenden Kalenderjahr vorangegangenen Jahren in anderen Journalen zitiert worden ist. Exakt definiert ist er als der Quotient aus der Zahl der in dieser Periode nachgewiesenen Zitate von Artikeln einer Zeitschrift und jener der in dieser Zeit dort veröffentlichten Arbeiten. Wenn also z. B.150 Zitate aufgefunden werden können

\author{
Key Words \\ Impact Factor · Source Journal · Scientometrics · Citation
}

\section{Summary}

The impact factor has become a widely used tool for the evaluation of scientific research. It is calculated by dividing the number of citations by the source items published in the journal during the previous two years. Despite its popularity, the parameter should be handled with care due to the many phenomena that influence citation rates. The correlation between the impact factor of a journal and the citation frequency of a single article is questionable. Author's selection of references is subject to factors unrelated to quality. There is a tremendous bias towards English language journals compared to those publishing in other languages. The impact factor favours research areas that promote short-term studies, whereas a tendency to treat long-term clinical investigations as less important is simultaneously created.

\begin{tabular}{ll}
\hline KARGER & @ 2000 S. Karger GmbH, Freiburg \\
$\begin{array}{l}\text { Fax }+497614520714 \\
\begin{array}{l}\text { E-mail Information@Karger.de } \\
\text { www.karger.com }\end{array}\end{array}$ & $\begin{array}{l}\text { Accessible online at: } \\
\text { www.karger.com/journals/onk }\end{array}$
\end{tabular}

und 50 Aufsätze erschienen sind, beträgt der Impact Factor 3 $[1,2]$. Die breite Datenbasis, die beständige Aktualisierung und die in der Vergangenheit mehrfach bewiesene prognostische Leistungsfähigkeit der Größe können und dürfen nicht bestritten werden. Viele Arbeiten der Spitzenforschung sind in Periodika mit hohem IF (z. B. «Lancet», «Science», «New England Journal of Medicine») erschienen. Der IF ist außerdem aus der gegenwärtigen Diskussion um wissenschaftliche Effizienz und deren Konsequenzen für Karrieren und Geschäfte nicht wegzudenken. Den Vorzügen steht allerdings eine Reihe von Schwächen gegenüber, die an seiner Gültigkeit und Zuverlässigkeit gewisse Zweifel wecken und zum Teil auch von seinen Urhebern eingestanden werden. Die folgenden zehn Thesen umreißen das Unbehagen am Impact Factor. 


\section{These 1: Die Messung wissenschaftlicher Leistungsfähigkeit ist weitgehend auf die Bibliometrie reduziert}

Mediziner haben die Szientometrie für das eigene Fachgebiet bisher als Metadisziplin betrachtet und dementsprechend kaum gepflegt. Die auf diesem Feld tätigen Soziologen, Psychologen und Informationswissenschaftler taxieren das akademische Ansehen vorrangig nach der Zahl der Vorträge, Veröffentlichungen und Patente. Diese Einschätzung kann dazu führen, daß die Bewertung wissenschaftlicher Leistung auf die Quantifizierung der Publikationstätigkeit reduziert wird, die ihrerseits von Zitierstatistiken beherrscht ist. Dadurch hat der IF die Chance erhalten, zu einem Surrogatmarker für wissenschaftliche Leistungsfähigkeit zu werden.

\section{These 2: Zitierstatistiken geben das wahre Zitierverhalten nur unvollständig wieder}

Die Entscheidung für bzw. gegen die Aufnahme eines wissenschaftlichen Aufsatzes in ein Literaturverzeichnis ist einer großen, weder allgemein noch im Einzelfall objektivierbaren Unsicherheit unterworfen. Es ist grundsätzlich unbewiesen, daß Wissenschaftler das und nur das zitieren, was sie bei der Planung und Durchführung der praktischen Arbeit und der Abfassung eines wissenschaftlichen Manuskripts beeinflußt hat. Im Einzelfall kann in den Text viel Material eingehen, das anderen verdankt und dessen Quelle dennoch nicht oder inkomplett genannt wird. MacRoberts und MacRoberts [3] glauben, daß nur etwa ein Drittel der in einem Manuskript versammelten externen Einflüsse durch Zitat kenntlich gemacht wird. Ein weiteres Drittel stellen nichtzitierte Anleihen; der Rest entstammt anderen Quellen. Aus den in der akademischen Medizin üblichen Literaturangaben wird auch nicht ersichtlich, ob der gesamte Aufsatz, ein Absatz oder lediglich ein Textfragment dem Leser bei den eigenen Forschungen und Formulierungen gedient hat.

\section{These 3: Der Impact Factor simplifiziert die Bewertung wissenschaftlicher Leistungen}

Zitate können positiv oder negativ intendiert sein, d.h., der Zitierende kann seine Zustimmung zu der veröffentlichten Meinung kundtun oder einen abweichenden Standpunkt vertreten. Gewiß wird mit dem überwiegenden Teil der Referenzen den zitierten Autoren Bestätigung und Anerkennung zuteil. Auch wenn eine zitierte Arbeit Kritik oder Auseinandersetzung auslöst, darf man sie als positiv bewerten. Falls dem Zitierten allerdings Flüchtigkeiten oder gar substantielle Fehler nachgewiesen werden, kann man sie ihm schwerlich gutschreiben. Die Identifizierung solcher Negativzitate fällt nicht leicht und ist vom subjektiven Urteil abhängig. Ihr Anteil wird jedoch auf $7 \%$ aller Zitate geschätzt [4]. Überdies bilden die Zitate den schwächsten, weil reproduktiv-komparativen Teil einer wissenschaftlichen Arbeit. Je mehr einer zitiert, um so weniger trägt er im allgemeinen Neues bei. Bestes Beispiel für diese Gattung wissenschaftlicher Publikationen sind die
Review-Artikel mit bis zu hundert Referenzangaben - und vielfach ebenso vielen Zitaten in der Nachfolgeliteratur.

\section{These 4: Die Strategie für die Vermarktung des Impact Factors schränkt seine Glaubwürdigkeit ein}

Das von Eugene Garfield 1958 gegründete Institute for Scientific Information (ISI) hat bis jetzt etwa 16000 sogenannte Quellenzeitschriften in seinen Fundus aufgenommen. Für den Bereich der Naturwissenschaften und der Medizin werden 4200 Periodika aus 80 Ländern ausgewertet. Nicht weniger als 97,4\% aller vom ISI gesammelten Zitate stammen aus englischsprachigen Arbeiten [5]. Die Werte für den IF bewegen sich zwischen einem Maximum von 50 und einem Minimum von 0,01 [6]. Die Daten des Instituts werden in «Current Contents», «Citation Index» und «Journal Citation Report» verbreitet. Das seit 1989 zusätzlich herausgegebene Nachrichtenblatt «Science Watch» enthält Rubriken mit effekthascherischen Titeln wie «Paper of the year», «Hottest research paper of 19..», «The top research institute in ... studies» und «The world's most cited scientist over the past decade».

\section{These 5: Der Impact Factor ist für die Beurteilung einzelner Autoren und Publikationen wenig geeignet}

Der IF soll strenggenommen ausschließlich der Reihung und dem Vergleich wissenschaftlicher Zeitschriften dienen. In der Praxis wird er jedoch mehr und mehr zur Bewertung und prognostischen Einschätzung der dort publizierenden Wissenschaftler herangezogen. Dagegen werden ernste Einwände erhoben [7]. Zum einen werden für seine Berechnung Artikel unzweifelhaft unterschiedlicher Qualität herangezogen, zum anderen werden am Schluß nicht die Werte des einzelnen Artikels oder einer einzelnen Nummer, sondern der in einem Zeitraum von 24 Monaten erschienenen Hefte gemittelt. Damit kann ein Autor unabhängig von den Zitaten, die er tatsächlich erhalten hat, den IF der Zeitschrift für sich beanspruchen. Im Extremfall wird ihm also der IF des Journals zugesprochen, auch wenn seine Arbeit nicht ein einziges Mal zitiert worden ist.

\section{These 6: Der Impact Factor begünstigt den Status quo der Wissenschaftspublizistik}

Ob ein Periodikum als Quellenzeitschrift anerkannt wird, entscheidet das Institut in Philadelphia. Die Auswahlkriterien sind überzeugend, begünstigen jedoch den Status quo. Für bisher nicht berücksichtigte Zeitschriften, insbesondere wenn sie neu auf den Markt gekommen sind, ist es außerordentlich schwer, in den Kreis der Source Journals aufgenommen zu werden. Nur Zitate aus etablierten Zeitschriften werden zur Berechnung des IF herangezogen. Die anderen - und sie stellen die große Masse der Publikationsorgane - können lediglich zitiert werden. 


\section{These 7: Die Sorge um den Impact Factor veranlaßt Herausgeber und Verleger zu strukturellen Eingriffen an den Journalen}

Um den Zähler in der Formel für den IF zu erhöhen, werden die Autoren von den Herausgebern aufgefordert, möglichst viele Arbeiten aus den beiden letzten Jahrgängen der eigenen Zeitschrift zu zitieren, auch wenn diese Zitate thematisch nur schwerlich zu rechtfertigen sind. Um den Nenner zu senken, werden kleine Beiträge, z.B. Kasuistiken, in einer äußeren Form publiziert, die sie von der Berücksichtigung als Originalarbeit ausnimmt. Langfristig werden Aufsätze mit hohem IFVorhersagewert, z. B. Review-Artikel, den Vorzug vor anderen Manuskripten erhalten. Man wird dann mancher Nummer ansehen können, daß sie nicht in erster Linie für den Käufer und Leser hergestellt, sondern auch und gerade mit besonderer Rücksicht auf den IF redigiert worden ist.

\section{These 8: Die Sorge um den Impact Factor verleitet Wissenschaftler und Autoren zu Abweichungen vom korrekten Zitierverhalten}

Die Konstruktion des IF macht es dem einzelnen Autor möglich, durch wiederholte Zitate eigener Arbeiten den persönlichen IF und jenen der Zeitschriften, in denen er publiziert, heraufzusetzen. $\mathrm{Zu}$ ähnlichen Verwerfungen der Referenzangaben führt die Bildung von Zitiergemeinschaften. Umgekehrt kann der IF durch Verzicht auf Zitate von Arbeiten konkurrierender Gruppen in anderer Richtung manipuliert werden. Als Faustregel sollte gelten, daß Eigenzitate nicht mehr als $10 \%$ der Referenzliste füllen.

These 9: Der Impact Factor berücksichtigt nur ungenügend die Unterschiede in der Dynamik der Forschung, die zwischen den verschiedenen Disziplinen bestehen

Arbeiten aus Grundlagen- und Querschnittsfächern werden mit Abstand am häufigsten zitiert. Die klinischen, insbesondere die sogenannten kleinen Fächer erzielen im Vergleich dazu nur geringe Zitierquoten. Dieser Unterschied erklärt sich zu einem großen Teil aus der Geschwindigkeit des fachspezi- fischen Wissensumsatzes. Man hat schon früh erkannt, daß der IF ohne facheigene Differenzierung und damit spezifische Bezugsgruppe im Einzelfall kaum repräsentativ ist. Es ist also in jedem Fall unabdingbar, daß die jeweilige Disziplin zusätzlich kenntlich gemacht wird.

These 10: Die Validierung des Impact Factors als
Gradmesser wissenschaftlicher Qualität steht aus

Die zum Teil mehrere Jahrzehnte alten Grundgesetze der Szientometrie sind durch die modernen Zitierstatistiken im wesentlichen bestätigt worden. Es fehlt jedoch weiterhin an einer einheitlichen Theorie des Zitierens im wissenschaftlichen Schrifttum. Die Zweifel an der Gültigkeit des IF werden so lange bestehen bleiben, wie die errechneten Werte glauben machen, daß sich die Journale an der Spitze und am Ende der Rangordnung in ihrer wissenschaftlichen Qualität um ein bis zwei Größenordnungen unterscheiden. Jeder wird für sein Fachgebiet konzedieren, daß Zeitschriften, die häufig zitiert werden, viel gelesen werden und daß sie deshalb viel gelesen werden, weil sie den Fortschritt der Wissenschaft mittragen. Der Umkehrschluß muß aber keineswegs in jedem Fall zutreffen.

\section{Schlußfolgerung}

Der forschende Mediziner pflegt die Gültigkeits- und Bewertungskriterien seiner Arbeit genau zu definieren und die methodischen Grenzen offenzulegen und zu diskutieren. An die Prüfung des IF sind die gleichen strengen Maßstäbe zu stellen. Die überzeugende Validierung des IF steht mangels eines vergleichbaren Standards freilich noch immer aus. Die Frage bleibt unbeantwortet, ob sich eine so umfangreiche Materie wie Forschungskapazität, Forschungsqualität und die Durchsetzung von Forschungsergebnissen durch die vergleichsweise simple Arithmetik des IF ausreichend erfassen läßt. Der Einwand gilt weniger für die Spitzenforschung, deren überragende Qualität auch ohne Berechnung des IF zu erkennen ist. Bei der überwiegenden Mehrzahl der Periodika und Autoren fallen die geschilderten Selektionsnachteile aber so stark ins Gewicht, daß die ermittelten Unterschiede nur mit großer Vorsicht gewertet werden dürfen.

\section{Literatur}

1 Garfield E: Citation indexing for studying science. Nature 1970;227:669-671.

2 Garfield E: Citation analysis as a tool in journal evaluation. Science 1972;178:471-479.

3 MacRoberts MH, MacRoberts BR: Citation count (Correspondence). Nature 1988;327:456.
4 Taubes G: Measure for measure in science. Science 1993;260:884-886.

5 Garfield E: Using the Impact Factor. Current Contents 1994;25:3-7.
6 Hansson S: Impact factor as a misleading tool in evaluation of medical journals (letter). Lancet 1995; 346:906.

7 Lindner UK, Oehm V: Die Magie des Impact Factors - Enttarnung eines Phänomens. Radiologe 1998; $38: 1-2$. 